Pacific Journal of Mathematics

INFINITE PRODUCTS OF SUBSTOCHASTIC MATRICES 


\section{INFINITE PRODUCTS OF SUBSTOCHASTIC MATRICES}

\section{N. J. Pullman}

This paper is about two types of infinite products of substochastic matrices $\left\{A_{j}\right\}$ namely: the left product defined by the sequence of left partial products $A_{1}, A_{2} A_{1}, A_{3} A_{2} A_{1}, \cdots$; and the right product defined by the sequence of right partial products $A_{1}, A_{1} A_{2}, A_{1} A_{2} A_{3}, \cdots$.

The basic theorem is that if the $A_{n}$ are each $\infty$ by $\infty$ then:

a. There is a nonempty set $E$ of substochastic sequences each of which (except possibly the zero sequence, 0 ) is the componentwise limit of a sequence of rows, one from each left partial product;

b. Any sequence $\left\{\rho_{n}\right\}$ of rows, one from each left partial product, can be approximated by a sequence of convex combinations $\left\{c_{n}\right\}$ of points of $E$ (that is, $\left\{\rho_{n}-c_{n}\right\}$ converges componentwise to the zero sequence), and $c . E=\{0\}$ if and only if every sequence of rows, one from each left partial product, converges to 0 .

Similar conclusions follow immediately for the right product of $\infty$ by $\infty$ doubly substochastic matrices.

The asymptotic behaviour of the right product of a special class of $\left\{A_{n}\right\}$ is also considered.

The finite case (that is, when all the $A_{n}$ are $r$ by $r$ ) for stochastic $A_{n}$ is treated independently for convenience, even though the result in this case (Theorem 1) is actually a direct consequence of the basic Theorem 1'. Its conclusion is that there is an $m$ by $r$ stochastic matrix $A$ with $1 \leqq m \leqq r$ and permutation matrices $Q_{n}$ such that

a. if $m<r$ then for some stochastic $r-m$ by $m$ matrices $C_{n}$ :

$$
\lim _{n \rightarrow \infty}\left\{A_{n} A_{n-1} \cdots A_{1}-Q_{n}\left(\begin{array}{c}
A \\
C_{n} A
\end{array}\right)\right\}=0
$$

and $b$. if $m=r$ then

$$
\lim _{n \rightarrow \infty}\left\{A_{n} A_{n-1} \cdots A_{1}-Q_{n} A\right\}=0 .
$$

Some results on fixed points are obtained in the finite case which carry over, in restricted form, to the infinite case.

A real matrix is said to be stochastic if none of its entries is negative and each of its row sums is 1 . Two types of infinite products which arise naturally from a given sequence $\left\{A_{n}\right\}$ of stochastic matrices are those whose $n$th partial products are $R_{n}=A_{1} A_{2} \cdots A_{n}$ and $L_{n}=$ $A_{n} A_{n-1} \cdots A_{1}$ respectively. We'll call the sequence $\left\{R_{n}\right\}$ the right 
product and the sequence $\left\{L_{n}\right\}$ the left product of the $A_{n}$.

The right product is of interest in the theory of Markov chains with possibly nonstationary transition probabilities because if $A_{n}$ is the matrix of probabilities $a_{i j}^{(n)}$ of transition from state $i$ at time $n-1$ to state $j$ at time $n$ then the $i j$ th entry $r_{i j}^{(n)}$ of $R_{n}$ is the probability of transition to state $j$ at time $n$ from state $i$ at time 0 .

The left product has a similar interpretation: $l_{i j}^{(n)}$ is the probability of transition from state $i$ at time $-n$ to state $j$ at time 0 .

We shall obtain theorems on the asymptotic behaviour of these partial products and on their fixed points. For example if the $A_{n}$ are $\infty$ by $\infty$ stochastic matrices we can show that there is a sequence of rows, one from each $L_{n}$, which converges componentwise.

The finite and infinite cases are treated separately for clarity.

Definition. A permutation matrix is a matrix of zeroes and ones which exactly one 1 in each row and each column.

THEOREM 1. If $L_{n}=A_{n} A_{n-1} \cdots A_{1}$ and each $A_{n}$ is an $r$ by $r$ stochastic matrix then there exists an $m$ by $r$ stochastic matrix $A$ with $1 \leqq m \leqq r, r$ by $r$ permutation matrices $Q_{n}$ and, if $m<r$, stochastic $r-m$ by $m$ matrices $C_{n}$ such that:

$$
\begin{aligned}
& \lim _{n \rightarrow \infty}\left\|L_{n}-Q_{n}\left(\begin{array}{c}
A \\
C_{n} A
\end{array}\right)\right\|=0 \quad \text { if } m<r \quad \text { and } \\
& \lim _{n \rightarrow \infty}\left\|L_{n}-Q_{n} A\right\|=0 \text { if } m=r .
\end{aligned}
$$

Proof. Let $S$ be the convex hull of the basis vectors $v_{1}=(1,0,0, \cdots, 0), v_{2}=(0,1,0, \cdots, 0), \cdots, v_{r}=(0,0,0, \cdots, 1)$. Each (S) $L_{n}$ is a convex polytope (that is, the convex hull of $p$ points), these polytopes are nested (that is, $(S) L_{n+1} \subseteq(S) L_{n}$ for all $n$ ) and none of them has more than $r$ vertices (a point $x$ of a polytope is a vertex if it is on no open line segment contained in the polytope). It can be shown that the intersection of such a family of convex polytopes is a convex polytope of $r$ or fewer vertices. Let $K=\bigcap_{n \geqq 1}(S) L_{n}$ and denote its vertices by $k_{1}, \cdots, k_{m}$. Let $A$ be the $m$ by $r$ matrix whose $i$ th row is $k_{i}$. Let $v_{i}^{(n)}$ denote $\left(v_{i}\right) L_{n}$. For each $n$ and each $t \leqq \mathrm{~m}$ there is a $v_{i_{t}}^{(n)}$ such that $k_{t}=\lim _{n \rightarrow \infty} v_{t_{t}}^{(n)}$. We can assume that for each $n$ there are only $m$ such $v_{i_{t}}^{(n)}$ so chosen. If $m<r$ extend the definition of $i_{t}$ so that $\left\{v_{i_{t}}^{(n)}: m<t \leqq r\right\}$ is the set of $v_{i}^{(n)}$ not already chosen. $Q_{n}$ is the matrix $\left(q_{i j}^{(n)}\right)$ for which $q_{i t}^{(n)}$ is 1 if $i=i_{t}$ and is 0 otherwise. If $m<r$ and $t>m$ let $k_{t}^{(n)}$ be the point of $K$ closest to $v_{t_{t}}^{(n)}$. Since $K$ is convex, $k_{t}^{(n)}$ is a convex combination, $\sum_{j=1}^{m} c_{t j}^{(n)} k_{j}$, of the vertices $K$. Therefore $C_{n}=\left(c_{2, j}^{(n)}\right)$ is an $r-m$ by $m$ stochastic matrix and $k_{t}^{(n)}=$ $\left(v_{t}\right)\left(\begin{array}{c}A \\ C_{n} A\end{array}\right)$ for each $m<t \leqq r$. Consequently $\left(v_{i_{t}}\right) Q_{n}\left(\begin{array}{c}A \\ C_{n} A\end{array}\right)=\left(v_{t}\right)\left(\begin{array}{c}A \\ C_{n} A\end{array}\right)$ if 
$m<r$ and $\left(v_{i_{t}}\right) Q_{n} A=\left(v_{t}\right) A$ if $m=r$. Theorem 1 then follows from the fact that $\lim _{n \rightarrow \infty} v_{i t}^{(n)}=k_{t}$ if $1 \leqq t \leqq m$ and $\lim _{n-\infty} v_{i_{t}}^{(n)}-k_{t}^{(n)}=0$ if $t>m$.

Notice that $\lim _{n \rightarrow \infty} L_{n}=\left(\begin{array}{c}k_{1} \\ k_{1} \\ \vdots \\ \dot{k}_{1}\end{array}\right)$ if $\mathrm{m}=1$ because $K$ then consists of the one vertex $k_{1}$.

DEFINITION. A sequence $\left\{P_{n}\right\}$ of $r$ by $r$ matrices is descending if and only if $(S) P_{n+1} \subseteq(S) P_{n}$ for all $n$ sufficiently large. ( $S$ is as in the proof of Theorem 1). As a first corollary to Theorem 1 we have: $m, Q_{n}, A$ and $C_{n}$ (if $m<r$ ) such that $\lim _{n \rightarrow \infty}\left\|P_{n}-Q_{n}\left(\begin{array}{c}A \\ C_{n} A\end{array}\right)\right\|=0$ if $m<r$ and $\lim _{n \rightarrow \infty}\left\|P_{n}-Q_{n} A\right\|=0$ if $m=r$, for all descending sequences because each such sequence (with the first $N$ terms omitted) is the left product of some sequence of stochastic matrices. (All left products of stochastic matrices are, of course, descending sequences.) Another immediate corollary concerns doubly stochastic matrices (that is, stochastic matrices whose transposes are also stochastic). We shall state the corollary emphasizing the matrix entries for variety's sake.

COROLLARY 2. If $\left\{A_{n}\right\}$ is a sequence of doubly stochastic $r$ by $r$ matrices and $R_{n}=A_{1} A_{2} \cdots A_{n}$ then there exists an $m$ by $r$ stochastic matrix $A$ with $1 \leqq m \leqq r$ and permutations $q_{n}$ of the $r$ indices such that for each $1 \leqq j \leqq r$ :

(a) if $1 \leqq q_{n}(i) \leqq m, \lim _{n \rightarrow \infty}\left(r_{j i}^{(n)}-a_{q_{n}(i) j}\right)=0$ and if $m<r$ there exist $r-m$ by $m$ stochastic matrices $C_{n}$ such that

(b) if $m<q_{n}(i) \leqq r$ then:

$$
\lim _{n \rightarrow \infty}\left(r_{j i}^{(n)}-\sum_{k=1}^{m} c^{(n)} q_{n}(i) k^{a} k_{j}\right)=0 .
$$

Some examples of $\left\{A_{n}\right\}$ with descending right products are provided by all those sequences of stochastic matrices $\left\{A_{n}\right\}$ which commute pairwise within a row permutation (i.e. $A_{n} A_{n^{\prime}}=Q_{n n^{\prime}} A_{n^{\prime}} A_{n}$ for some permutation matrix $Q_{n n^{\prime}}$ ). Because of their connection with Markov chains we shall investigate descending right products further. We'll impose further conditions on the $A_{n}$ which are not too stringent but which give additional information about the $C_{n}$ of Theorem 1 . While doing so we acquire some information on the fixed points of $A_{n}$ and $R_{n}$.

Definition. $B$ occurs frequently among the $A_{n}$ if and only if $B=A_{n}$ for infinitely many $n$.

Lemma. If $\left\{A_{n}\right\}$ is a sequence of $r$ by $r$ stochastic matrices whose 
right partial products $R_{n}=A_{1} A_{2} \cdots A_{n}$ are a descending sequence and $B$ occurs frequently among the $A_{n}$ then, in the notation of Theorem 1 there is an $m$ by $m$ permutation matrix $D$ such that $A B=D A$.

Proof. For some $N,\left\{R_{N+n}\right\}$ is the left product of some sequence of stochastic matrices $A_{n}^{\prime}$. Let $K$ be as in the proof of Theorem 1 applied to the $A_{n}^{\prime}$. Then $K=\bigcap_{n>N}(S) R_{n}$. $(K) B \cong K$ because $K=$ $\bigcap\left\{(S) R_{n-1}: B=A_{n}\right.$ and $\left.n>N\right\}$. Suppose $x \in K$. Then, for infinitely many $n$, there are $x_{n} \in(S) R_{n-1}$ for which $x=\left(x_{n}\right) B$. A subsequence $\left\{x_{n_{m}}\right\}$ converges to some point $y \in S$. Therefore $\left(x_{n_{m}}\right) B$ converges to $(y) B$ and hence $x=(y) B$. But $y \in K$ and hence $K \subseteq(K) B$. Thus $K=(K) B$ and hence $B$ permutes the vertices of $K$ (rows of $A$ ). Let $D$ be the $m$ by $m$ permutation matrix representing this row permutation then $A B=D A$.

$B$ permutes all the vertices of $K$ and fixes the barycentre, $1 / m^{\prime} \sum_{t=1}^{m^{\prime}} k_{i_{t}}$, of each subset $\left\{k_{i_{1}}, k_{i_{2}}, \cdots, k_{i_{m}}\right\}$ of $m^{\prime}$ vertices of $K$ (rows of $A$ ) which it permutes. Therefore $(x) B=x$ for all $x$ in the convex hull of these barycentres. There may be (left) fixed points of $B$ outside the convex hull of the barycentres.

Let us enumerate all the matrices occurring frequently among the $A_{n}$ so that $A_{n_{1}}$ is the first such matrix and $A_{n_{p}}$ is the $p$ th such matrix distinct from $A_{n_{p}-1}$. Let $D_{n_{p}}$ be the $m$ by $m$ permutation matrix corresponding to $A_{n_{p}}$ (as in the lemma) and let $D_{n}=D_{n_{p}}$ if $A_{n}=A_{n_{p}}$. Applying the lemma to the first corollary to Theorem 1 we obtain:

THEOREM 2. If $\left\{A_{n}\right\}$ is a sequence of $r$ by $r$ stochastic matrices each of which (except for finitely many $n$ ) occur frequently among the $A_{n}$ and the $n$-th partial products $R_{n}=A_{1} A_{2} \cdots A_{n}$ are descending then there exists an $m$ by $r$ stochastic matrix $A$ (with $1 \leqq m \leqq r$ ), permutation matrices $Q_{n}$ and, if $m<r, r-m$ by $m$ stochastic matrices $C_{n}$ such that given $\varepsilon>0$ there is an $N$ for which:

$$
\begin{aligned}
& \text { (a) }\left\|R_{n}-Q_{N}\left(\begin{array}{c}
D_{n}^{\prime} A \\
C_{N} D_{n}^{\prime} A
\end{array}\right)\right\|<\varepsilon \quad(\text { if } m<r), \\
& \text { (b) }\left\|R_{n}-Q_{N} D_{n}^{\prime} A\right\|<\varepsilon \quad(\text { if } m=r),
\end{aligned}
$$

for all $n>N$. $D_{n}^{\prime}$ is the permutation matrix which is the product $D_{N+1} D_{N+2} \cdots D_{n}$ of $D_{s}$ defined in the previous paragraph. Moreover the barycentres of those sets of rows of $A$ which are permuted by all the $D_{n_{p}}$ is a (left) fixed point for all $A_{n}$ (except perhaps the finitely many $n$ for which $A_{n}$ does not occur frequently). In particular the barycentre $b=1 / m \sum_{i=1}^{m}\left(a_{i 1}, \cdots, a_{i r}\right)$ of the rows of $A$ is such a (left) fixed vector.

Let $F$ be the convex hull of the barycentres mentioned in Theorem 
2. $F$ is fixed (pointwise) by each of those $A_{n}$ which occur frequently. If all the $A_{n}$ occur frequently then $(x) R_{n}=x$ for all $n$ and all $x \in F$.

The fundamental theorems on the convergence of the powers of the transition matrix and the "classification of states" of a finite Markov chain with stationary transition probabilities (see for example [4] pp. 170-184) can be obtained from Theorem 1 by examination of the position of $K$ in $S$. In the interest of brevity we shall not do so here but shall instead discuss two notions from the stationary case by way of sample applications of Theorems 1 and 2 .

In the notation of the proof of Theorem 1 let $T$ be the set of all $i$ for which $v_{i}$ is not in the set of basis vectors spanning $K$. Following the custom (see e.g. [2]) for the stationary case we'll say that $i$ leads to $j$ (written $i \sim^{x} j$ ) if and only if $r_{i j}^{(n)}>0$ for some $n$. If the right product of the $A_{n}$ is descending then for each $i, \lim _{n \rightarrow \infty} r_{i j}^{(n)}=0$ for all $j \in T$ and; each $i \in T$ leads to some $j \notin T$ by the first corollary to Theorem 1. In the stationary case (i.e. when $A_{n}=A_{1}$ for all $n$ ):

$$
T=\bigcup_{j \geqq 1}\left\{i: i \sim^{x} j \text { and } j \chi^{x} i\right\} \text {. }
$$

This is precisely the definition of the set of transient (sometimes called inessential) states in the stationary case.

The notion of regular chain (in the terminology used in [6]) can be extended to the nonstationary case so as to obtain the same kind of basic result. Suppose the right product of the $A_{n}$ is descending and that there is a product $P=A_{n_{p_{1}}} A_{n_{p_{2}}} \cdots A_{n_{p_{q}}}$ of frequently occuring $A_{n_{p_{q_{6}}}}$ (in the notation of Theorem 2) which is positive (i.e. $p_{i j}>0$, all $i, j$ ). (The $n_{p_{i}}$ are not necessarily distinct nor in increasing order). Call such $\left\{A_{n}\right\}$ regular sequences. It then follows that the right products $R_{n}$ of regular sequences $\left\{A_{n}\right\}$ converge to a matrix all of whose rows are the vector $k$. No component of $k$ is zero, $(k) R_{n}=k$ for all sufficiently large $n$ (for all $n$, if $(S) R_{n+1} \subseteq(S) R_{n}$ for all $n$ ) and $k$ is the only vector in $S$ with this property. Although this is equivalent to the corresponding result for the stationary case it is easy enough to obtain using the first corollary to Theorem 1 and the lemma preceding Theorem 2: All we need do is show that $m=1$. To this end observe that according to the lemma, $P$ permutes the vertices of $K$ so that, for some $n$ : (x) $P^{n}=x$ for all $x \in K$. If $K$ had more than one vertex the line joining two of them would meet the boundary of $S$ in a point $x$ which is fixed by $P^{n} . \quad(x) P^{n}$ can have no zero components because $P$ is positive but $x$ has zero components because it's in the boundary of $S$. This second application may also be found in a slightly less general form as Theorem 3 of [5].

Definition. A real matrix is substochastic if and only if none of 
its entries is negative and 1 is an upper bound for its row sums.

Most of the foregoing results including Theorem 1 and its corollaries can be extended to infinite as well as finite substochastic matrices. To do so, consider the set $S_{0}$ of all substochastic sequences (i.e. the set of all real sequences of nonnegative terms whose sum is at most 1). $S_{0}$ is a compact, convex subset of the space of all real sequences under the product topology. The $\infty$ by $\infty$ substochastic matrices are associative and closed under matrix multiplication so that left and right product is defined for every sequence of such matrices.

THEOREM 1'. If $\left\{L_{n}\right\}$ is the left product of a sequence of $\infty$ by $\infty$ substochastic matrices then there is a nonempty set, $E$, of substochastic sequences with the following properties:

(a) For each $k \in E$ (except possibly the zero sequence) and each $n$ there is an integer $i_{n, k}$ such that for all $j$ :

$$
\lim _{n \rightarrow \infty} l_{i_{n, k^{j}}^{(n)}}=k_{j} \text {. }
$$

(b) For each sequence $\left\{i_{n}\right\}$ there is a convex combination $x^{(i, n)}$ of elements of $E$ such that for all $j$ :

$$
\lim _{n \rightarrow \infty}\left(l_{i_{n} j}^{(n)}-x_{j}^{(i, n)}\right)=0 \text {. }
$$

(c) The zero sequence is the only element of $E$ if and only if for all sequences $\left\{i_{n}\right\}$ and all $j$ :

$$
\lim _{n \rightarrow \infty} l_{i_{n} j}^{(n)}=0
$$

Proof. For each subset $F$ of $S_{0}$ let $c o(F)$ be the set of convex combinations of elements of $F$ and $\overline{c o}(F)$ be the intersection of all closed convex sets containing $F$. Let $W_{n}$ be the set consisting of 0 and all the rows of $L_{n}$, let $\bar{L}_{n}=\overline{c o}\left(W_{n}\right)$ and $K=\bigcap_{n>1} \bar{L}_{n} . \quad K$ is convex and compact and $0 \in K$. Let $E$ be the set of extremals of $K$ (that is, $k \in E$ if and only if $k \in K$ and $k$ is an interior point of no line segment in $K$ ) then $K=\overline{c o}(E)$ by the Krein-Milman theorem. Part (a) of Theorem $1^{\prime}$ is proven by contradiction. Suppose $k \in E$ and a neighbourhood of $k$ excludes 0 and all rows of $L_{n}$ for all $n$ in an infinite set $\Omega$. Then, for a finite set $\Lambda$ and some $\varepsilon>0, W_{n}$ is in the complement of $Z \equiv \bigcap_{j \in A}\left\{x \in S_{0}:\left|x_{j}-k_{j}\right|<\varepsilon\right\}$ for each $n \in \Omega$.

Let $T_{j}^{+}=\left\{x \in S_{0}: x_{j} \geqq k_{j}+\varepsilon\right\}, \quad T_{j}^{-}=\left\{x \in S_{0}: x_{j} \leqq k_{j}-\varepsilon\right\} \quad$ and $T_{j}=T_{j}^{+} \cup T_{j}^{-}$. Then

$$
\begin{aligned}
K \subseteq \bar{L}_{n} & =\overline{c o}\left(\bigcup_{j \in A}\left(T_{j} \cap W_{n}\right)\right) \\
& =c o\left(\bigcup_{j \in A} \overline{c o}\left(T_{j} \cap W_{n}\right)\right)
\end{aligned}
$$




$$
\begin{aligned}
& =c o\left(\bigcup_{j \in A} \overline{c o}\left(\left(T_{j}^{+} \cap W_{n}\right) \cup\left(T_{j}^{-} \cap W_{n}\right)\right)\right) \\
& =c o\left(\bigcup_{j \in A} \operatorname{co}\left(\overline{c o}\left(T_{j}^{+} \cap W_{n}\right) \cup \overline{c o}\left(T_{j}^{-} \cap W_{n}\right)\right)\right)
\end{aligned}
$$

(again by [3] V 2.5)

$$
\begin{aligned}
& \cong \operatorname{co}\left(\bigcup_{j \in \Lambda} \operatorname{co}\left(\left(T_{j}^{+} \cap \bar{L}_{n}\right) \cup\left(T_{j}^{-} \cap \bar{L}_{n}\right)\right)\right) \\
& \subseteq \operatorname{co}\left(\bigcup_{j \in A} \operatorname{co}\left(T_{j} \cap \bar{L}_{n}\right)\right) .
\end{aligned}
$$

If $U_{j n}=T_{j} \cap \bar{L}_{n}$ is empty for some $j \in \Lambda, n \in \Omega$ then $U_{j m}=\phi$ for all sufficiently large $m$ because the $U_{j_{n}}$ are nested for fixed $j$. Rather than change notation, we can assume that $U_{j n} \neq \phi$ for all $n \in \Omega$ and all $j \in \Lambda$. Thus $k$ is a convex combination, $\sum_{j_{\in A}} \lambda_{j_{n}} u_{j_{n}}$, of elements $u_{j_{n}}$ of $c o\left(U_{j n}\right) . \quad U_{j_{n}}$ is the union of $U_{j n}^{+}=T_{j}^{+} \cap \bar{L}_{n}$ and $U_{j_{n}}^{-}=T_{j}^{-} \cap \bar{L}_{n}$. Assuming first that $U_{j n}^{+}$and $U_{-j n}^{-}$are nonempty for all $n \in \Omega$ we have $0 \leqq \mu_{j_{n}} \leqq 1$ such that $u_{j_{n}}=\mu_{j_{n}} u_{j n}^{+}+\left(1-\mu_{j_{n}}\right) u_{j_{n}}^{-}$for some $u_{j_{n}}^{+} \in U_{j_{n}}^{+}$and some $u_{\overline{j n}} \in U_{\overline{j n}}$. By successive extraction of subsequences we obtain $u_{j}^{+}$, $u_{j}^{-}, \mu_{j}$ and $\lambda_{j}$ such that

$$
\begin{array}{ll}
\lim _{m \rightarrow \infty} u_{j n_{m}}^{+}=u_{j}^{+}, & \lim _{m \rightarrow \infty} u_{j n_{m}}^{-}=u_{j}^{-}, \quad \lim _{m \rightarrow \infty} \mu_{j n_{m}}=\mu_{j}, \\
\lim _{m \rightarrow \infty} \lambda_{j n_{m}}=\lambda_{j}, & 1 \geqq \mu_{j} \geqq 0, \quad 1 \geqq \lambda_{j} \geqq 0 \text { and } \sum_{j \in A} \lambda_{j}=1 .
\end{array}
$$

Therefore $\left.k=\sum_{j \in A} \lambda_{j}\left(\mu_{j} u_{j}^{+}+\left(1-\mu_{j}\right) u_{j}^{-}\right)\right)$, and for all $j \in \Lambda: u_{j}^{+}, u_{j}^{-} \in K$ and $u_{j}^{+}, u_{j}^{-} \in T_{j}$. The extremality of $k$ implies that $k=u_{j}^{+}$or $u_{j}^{-}$for some $j$ and hence that $k \in T_{j}$. Consequently $k \notin Z$, a contradiction. If, however, $U_{j n}^{+}$or $U_{j n}^{-}$is $\phi$ for some (and hence all subsequent) $n$ we can use a similar argument using the $u_{j_{n}}$ instead of the $u_{j n}^{+}$and $u_{j n}^{-}$.

If $k \neq 0$ we can therefore assert that each sufficiently small neighbourhood of $k$ excludes 0 but contains an element of $W_{n}$ for all sufficiently large $n$. These elements must be rows of the $L_{n}$. Therefore $k$ is the componentwise limit of a sequence of rows, one from each $L_{n}$.

To prove part (b) let $d$ be the metric on $S_{0}$ which induces the product topology (see [1] II prop. 6, p. 97). Let $y_{n} \in L_{n}$ and $z_{n}$ be a point of $K$ closest to $y_{n}$ in the metric. $d\left(z_{n}, y_{n}\right)$ is a null sequence because the $\bar{L}_{n}$ are nested. A sequence $\left\{x_{n}\right\}$ in $c o(E)$ can be found for which $d\left(x_{n}, y_{n}\right)$ is a null sequence because $c o(E)$ is dense in $\overline{c o}(E)$ (see [3] V 2.4). Part (b) then follows if the $i_{n}$ th row of $L_{n}$ is used for $y_{n}$. Part (c) follows directly from parts (a) and (b). This completes the proof of Theorem 1'.

The conclusion of Theorem $1^{\prime}$ is valid if $\left\{L_{n}\right\}$ is replaced by any descending sequence $\left\{P_{n}\right\}$ of $\infty$ by $\infty$ substochastic matrices using the previous definition of "descending" with $S$ replaced by $S_{0}$. Such se- 
quences too are, except for finitely many terms, the left product of some sequence of substochastic matrices.

The statements about commutivity also carry over to the infinite case.

Corollary 2 extends to:

COROLLARY 2'. If $\left\{R_{n}\right\}$ is the right product of $\infty$ by $\infty$ doubly stochastic matrices then there is a nonempty set, $E$, of substochastic sequences with the following properties:

(a) For each non-zero $k \in E$ and each $n$ there is an integer $i_{n, k}$ such that for all $j$ :

$$
\lim _{n \rightarrow \infty} r_{j i_{n, k}}^{(n)}=k_{j} \quad \text { and }
$$

(b) For each sequence $\left\{i_{n}\right\}$ there is a convex combination $x^{(i, n)}$ of elements of $E$ such that for all $j$ :

$$
\lim _{n \rightarrow \infty} r_{j i_{n}}^{(n)}-x_{j}^{(i, n)}=0,
$$

(c) The zero sequence is the only element of $E$ if and only if for all $\left\{i_{n}\right\}$ and for all $j$ :

$$
\lim _{n \rightarrow \infty} r_{j i_{n}}^{(n)}=0
$$

A substochastic matrix is continuous on $S_{0}$ if and only if all of its columns are null sequences. If a continuous $B$ occurs frequently among the $A_{n}$ and their right product is descending then $(K) B=K$.

Theorem 2 and the remarks following it concerning fixed points also hold for $\infty$ by $\infty$ substochastic matrices $A_{n}$ provided each $A_{n}$ is continuous and $K$ has only finitely many extremals.

\section{REFERENCES}

1. N. Bourbaki, Eléments de mathématiques, Livre V, Espaces vectoriel topologiques, Hermann, Paris, 1942.

2. K. L. Chung, Markov Chains with Stationary Transition Probabilities. Springer, Berlin, 1960.

3. N. Dunford and J. Schwartz, Linear Operators, Part I, Interscience, New York 1958.

4. J. L. Doob, Stochastic Processes, Wiley, New York, 1953.

5. J. Hajnal, The ergodic properties of non-homogeneous finite Markov chains, Proc. Camb. Phil. Soc. 52 (1956), 67-77.

6. J. Kemeny and J. Snell, Finite Markov Chains, Van Nostrand, New Jersey 1960. 


\section{PACIFIC JOURNAL OF MATHEMATICS}

\section{EDITORS}

\author{
H. SAMmLSON \\ Stanford University \\ Stanford, California \\ R. M. BLUMENTHAL \\ University of Washington \\ Seattle, Washington 98105
}

\author{
*J. DUGUNDJI \\ University of Southern California \\ Los Angeles, California 90007
}

RICHARD ARENS

University of California

Los Angeles, California 90024

\section{E. F. BECKENBACH \\ B. H. NEUMANN \\ ASSOCIATE EDITORS}

\section{SUPPORTING INSTITUTIONS}

\author{
UNIVERSITY OF BRITISH COLUMBIA \\ CALIFORNIA INSTITUTE OF TECHNOLOGY \\ UNIVERSITY OF CALIFORNIA \\ MONTANA STATE UNIVERSITY \\ UNIVERSITY OF NEVADA \\ NEW MEXICO STATE UNIVERSITY \\ OREGON STATE UNIVERSITY \\ UNIVERSITY OF OREGON \\ OSAKA UNIVERSITY \\ UNIVERSITY OF SOUTHERN CALIFORNIA
}

\author{
STANFORD UNIVERSITY \\ UNIVERSITY OF TOKYO \\ UNIVERSITY OF UTAH \\ WASHINGTON STATE UNIVERSITY \\ UNIVERSITY OF WASHINGTON \\ * * * \\ AMERICAN MATHEMATICAL SOCIETY \\ CHEVRON RESEARCH CORPORATION \\ TRW SYSTEMS \\ NAVAL ORDNANCE TEST STATION
}

\footnotetext{
Mathematical papers intended for publication in the Pacific Journal of Mathematics should be typewritten (double spaced). The first paragraph or two must be capable of being used separately as a synopsis of the entire paper. It should not contain references to the bibliography. Manu. scripts may be sent to any one of the four editors. All other communications to the editors should be addressed to the managing editor, Richard Arens at the University of California, Los Angeles, California 90024.

50 reprints per author of each article are furnished free of charge; additional copies may be obtained at cost in multiples of 50 .
}

The Pacific Journal of Mathematics is published monthly. Effective with Volume 16 the price per volume (3 numbers) is $\$ 8.00$; single issues, $\$ 3.00$. Special price for current issues to individual faculty members of supporting institutions and to individual members of the American Mathematical Society: $\$ 4.00$ per volume; single issues $\$ 1.50$. Back numbers are available.

Subscriptions, orders for back numbers, and changes of address should be sent to Pacific Journal of Mathematics, 103 Highland Boulevard, Berkeley 8, California.

Printed at Kokusai Bunken Insatsusha (International Academic Printing Co., Ltd.), No. 6, 2-chome, Fujimi-cho, Chiyoda-ku, Tokyo, Japan.

PUBLISHED BY PACIFIC JOURNAL OF MATHEMATICS, A NON-PROFIT CORPORATION

The Supporting Institutions listed above contribute to the cost of publication of this Journal, but they are not owners or publishers and have no responsibility for its content or policies.

* Paul A. White, Acting Editor until J. Dugundji returns. 


\section{Pacific Journal of Mathematics}

\section{Vol. 16, No. $3 \quad$ BadMonth, 1966}

Gert Einar Torsten Almkvist, Stability of linear differential equations with

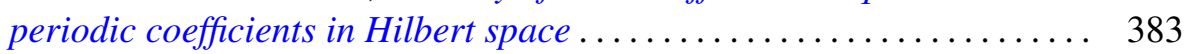

Richard Allen Askey and Stephen Wainger, A transplantation theorem for ultraspherical coefficients ................................ 393

Joseph Barback, Two notes on regressive isols .................. 407

Allen Richard Bernstein and Abraham Robinson, Solution of an invariant subspace problem of K. T. Smith and P. R. Halmos .............. 421

P. R. Halmos, Invariant subspaces of polynomially compact operators . . . . 433

Leon Bernstein, New infinite classes of periodic Jacobi-Perron algorithms.................................... 439

Richard Anthony Brualdi, Permanent of the direct product of matrices .... . 471

W. Wistar (William) Comfort and Kenneth Allen Ross, Pseudocompactness and uniform continuity in topological groups .................. 483

James Michael Gardner Fell, Algebras and fiber bundles . . . . . . . . . . . . 497

Alessandro Figà-Talamanca and Daniel Rider, A theorem of Littlewood and lacunary series for compact groups ..................... 505

David London, Two inequalities in nonnegative symmetric matrices...... 515

Norman Jay Pullman, Infinite products of substochastic matrices ........ 537

James McLean Sloss, Reflection and approximation by interpolation along

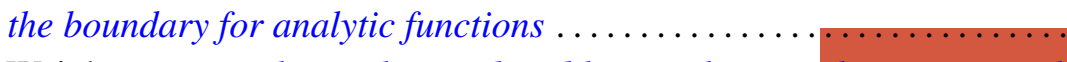

Carl Weinbaum, Visualizing the word problem, with an application to sixth groups................................... 